\title{
Arms Race Within Information Ecosystems ${ }^{1}$
}

\author{
Bengt Carlsson, Rune Gustavsson \\ Blekinge Institute of Technology, 37125 Ronneby, Sweden \\ e-mail: bengt.carlsson@bth.se, rune.gustavsson@bth.se
}

\begin{abstract}
Interacting agents of exploiters and users within an information ecosystem may be regarded both as biological beings and as part of an economic system of infohabitants. A protection system can be implementing as a filter governing the access to assets. Typically we will have a chain of attacks and countermeasures concerning this access to the desired assets. We model this process as an arms race. We base our model on microeconomics and a process model of a protection system based on exposure time. A user's reaction against an exploiter measure could either be a direct response to the measure or trying to anticipate future attacks by more general means of defeating the protection of the exploiter agent. When anticipating future attacks and countermeasures, both users and exploiters will improve their methods and tools due to an arms race. Our arms race model refines the competition as modeled in computational markets to model aspects which typically arise when societies grows beyond what can be controlled in a centralized manner. A dynamic, evolving and robust ecosystem of autonomous agents is a preferred and possible outcome of the arms race.
\end{abstract}

\section{Background}

Information ecosystems are often modeled as societies of agents, that is, Multi Agent Systems (MAS). A key aspect of MAS is coordination of tasks and activities. Distribution of subtasks and coordination of team activities has been a focus of MAS and DAI (Distributed AI) R\&D since early 1980's [3,8]. Since the end of 1980's R\&D in MAS has also addressed larger sets, societies, of agents. Issues such as articulation work, team formation and contracts as well as societal aspects of obligations, norms and social order are topics of present day research agendas $[4,6$, 10]. Of specific concerns are issues of resource allocations.

With computations markets we can model certain aspects of coordination in agent societies. The introduction of a common exchangeable commodity of 'money' and an efficient mechanism for settling the market value has turned out to be very fruitful. However, the market mechanism does not cover all aspects of society behavior related

\footnotetext{
${ }^{1}$ This work is partly supported by the European Project ALFEBIITE (A Logical Framework for Ethical Behavior between Infohabitants in the Information Trading Economy of the Universal Information Ecosystem): IST-1999-10298.
}

CIA-finala submitted to CIA workshop : 2001-11-19 : 09:41 
to coordination. In large and open societies as anticipated in information ecosystems and witnessed on the web we have to address other aspects as well. In order to model explicit non-friendly activities that can be seen on the web today such as tampering, cyber crime, and parasitic behavior, we introduce a arms-race model of coordination based on a dynamic model of security and an evolutionary model [2,7,11].

Our arms-race model refines the competition as modeled in computational markets to model aspects which typically arise when societies grows beyond what can be controlled in a centralized manner and coordination also has to include protective or 'hardening' mechanisms for the survival of 'computational species'.

Time is an important factor when adapting a successful behavior. Fitness or money as a measurement of estimated resources may be seen as another way of describing the amount of time needed. The purpose for doing this reduction of an economic system is to emphasis on conflict resolution within information ecosystem.

Within a developed economy there will be an exchange of value between infohabitants. A "fair" exchange, a use-value $U$, based on calculating the proper amount of resources needed for the exchangeable good can be settled. But nothing prevents infohabitants to be more selfish than just fair in their actions. As a matter of fact, this extra incentive is a necessarily ingredient in an evolving economic system. Let us distinguish two groups of infohabitants; the exploiter and the user agents, both groups controlled by humans.

The reason for an exploiter to manipulate a user is to get a surplus value, or profit, $S$. Instead of the ideal use-value $U$ there is a higher total price $T$, its exchangevalue, expressed as:

$$
T=U+S
$$

It is in the exploiters interest to make $S$ as big as possible. We claim that within information ecosystem the dynamics between exploiters and users can be modeled as an arms race focusing on the protection and access of assets. An improvement of the protection by an exploiter starts a counter-measure by users or other exploiters. Because of the increased competition between the antagonistic groups and because of the autonomous individuals within a group, an accelerating arms race may also improve the overall robustness of the ecosystem. Within biology arms race is often used to explain the origin of robust ecosystems. The success of a single infohabitant may be favored by coalitions and vigilance against new intruders.

In an information ecosystem the exploiter agents may be a useful part of a manufacturer production line making extra profit, or belong to humans with malicious intentions. These different roles of an exploiter agent are sometimes hard to separate. Malicious intentions include agents creating viruses or cause information trespass. Some users may regard a company having agents that send spam mails or spywares, as malicious. Response agents' form on the user side acts as a defense against exploiters and their agents.

Together these different agent societies constitute the participants in an arms race. Advanced tools for both exploiting and creating responsive actions by the different societies will be developed. In the ecosystem it will be hard to predestinate which tool that will be used by one or another agent. Exploiters and users evolve a dynamic fashion based on arms race within a refined set of exploiter and defense agents. The 
success will be determined by the reaction time against a certain attack by an agent and the strength of the attack versus the strength of the protection system. We will, in the next section outline a generic model of arms race.

\section{A Model of Arms Race}

We begin by defining a generic computational model of Arms Race based on microeconomics. Based on this model, we outline an Arms Race model based on dynamics of a protection system.

Our model is based on balancing supply-demand of an asset (good) in a market. In micro-economy we can calculate (e.g., by auctions) the ideal price, use-value $U$ above, of a good given the supply and demand. However, an exploiter can take advantage of different constraints or policies to ask for and get a higher price (adding extra profit or generating surplus value $S$ ) in accordance with eq. (1). above. Some well-known surplus enablers are: copyrights and patents, distribution restrictions, regulations, and added value. These surplus value mechanisms can be enforced in a computational Ecosystem by implementing 'filtering mechanisms' protecting assets by controlling access. In this paper we focus on a suitable dynamic protection model of access control.

An exploiter wants to protect surplus enabling mechanisms to his advantage, or to weaken those of his competitor. A user wants to get rid of those extra costs of getting the good. A user can thus legally or illegally attack suitable surplus generating mechanisms. Other exploiters can also enforce a user's intentions in a hostile takeover attempt.

The protection of the assets is based in interactions between three components. These components are a protection mechanism $P$, a detection mechanism $D$, and a response mechanism $R$. An implementation of those components acts as a 'filter' protecting the access to the assets. The filter is engineered to meet a class of attacks, $a$, it has a certain strength $s$, which can be improved, and it is time dependent, $t$. Our model is based on a model, Time Based Security, in network security [9]. An extended model [5] gives a process model of general protection systems. Let $\Delta P$ denote the duration of protection of a system at an attack $a$ starting at time $t_{0}$ and with protection strength $s$. Let $\Delta D$ denotes the time it take to detect an attack $a$ during the given circumstances. Finally, let $\Delta R$ denote the time it takes to implement sufficient measures to eliminate the threats of the attack $a$ after the attack has been detected. Eq. (2). captures the relations between those time intervals and introduces an exposure time $\Delta E\left(a, s, t_{o}, t\right)$, where $t>t_{0}$. The protection system is protecting the surplus granting mechanisms if and only if $\Delta E\left(a, s, t_{o}, t\right)$ is non-positive. If the exposure time $\Delta E\left(a, s, t_{o}, t\right)$ is positive the exploited part will have full control of the situation during that time interval. In all realistic situations $\Delta P\left(a, s, t_{o}, t\right)=0$ for all $t$ larger than a certain value.

CIA-finala submitted to CIA workshop : 2001-11-19: 09:41 


$$
\Delta E\left(a, s, t_{0}, t\right)=\Delta D\left(a, s, t_{0}, t\right)+\Delta R\left(a, s, t_{0}, t\right)-\Delta P\left(a, s, t_{0}, t\right)
$$

The eq. (2). may be used for proposing a surplus value formula based on eq. (1). The behavior of antagonistic agents within information ecosystems is discussed in [1]. The idea behind linking together a protection formula with surplus values is the extra cost these exploiter agents causes the users.

We can now formulate the total profit or surplus value for the exploiter, given that the protection system will stand against all attacks during the time interval 0 to $\mathrm{T}$, as eq. (3). below. The function $f$ is non-negative and eventually zero (e.g., when copyrights or patents ends).

$$
S=\int_{0}^{T} f(a, s, \tau) d \tau
$$

Eq. (2). includes all kinds of protections. It could be a security attack sending viruses or denial of service messages, but as well an economic based attack sending spam mails or using spy-wares. All activities are supposed to involve some advantage, the surplus value, for the exploiter behind the protection system.

Arms race can be modeled as follows. If we have a successful attack, i.e., $\Delta E>0$ for $t>t_{1}$, where $t_{1}$ denotes a point in time and $t_{1}>t_{0}$, then the exploiting agent can estimate a loss $L$ according to eq. (4). The function $h$ is non-negative and includes not only estimated loss of surplus value but also loss of, e.g., reputation.

$$
L(t)=\int_{t_{1}}^{t} h(a, s, \tau) d \tau
$$

The exploiter can decide to take countermeasures to bring back the favorable situation of a non-positive $\Delta E$ at some later time than $t_{1}$. This decision will be based on eq. (4). and an assessment of potential future profits based on eq. (3). The positive countermeasure, from the exploiters point of view, is modeled as an increase in strength (from $S$ to $S_{1}, S_{1}>S$, in the formulas above). Hence after a new point in time $t_{2}, t_{2}>t_{1}$, we will again have a non-positive $\Delta E$ for some time $t>t_{2}$.

The endurance $F$ of the exploiter during the time interval $\left[0, \mathrm{t}_{2}\right]$ is captured by eq. (5). The functions $g_{i}$ models the actual losses to the exploiter due to active users exploiting the breakthrough of the protection system. Other exploiters aiming at building up opportunities for generating surplus for them can facilitate the spread of the knowledge of the breakthrough (i.e., increase the cardinality of index I).

$$
F\left(t_{1}, t_{2}\right)=\int_{0}^{t_{1}} f(a, s, \tau) d \tau-\sum_{i \in I} \int_{t_{1}}^{t_{2}} g_{i}(a, \tau) d \tau-C_{s_{1}}
$$


The constant $C_{s_{1}}$ denotes the cost of strengthening the system from $\mathrm{s}$ to $\mathrm{s}_{1}$. The endurance, i.e., the value of $F\left(t_{1}, t_{2}\right)$ has to be positive for the exploiter to remain competitive.

An arms race can in our scenario be modeled as the sequence of change in sign of $\Delta \mathrm{E}$ of eq. (2). This changing in sign induces a sequence of time points $\left\{\mathrm{t}_{0}, \mathrm{t}_{1}, ..\right\}$ and a sequence of strengths of the protection system $\left\{\mathrm{s}, \mathrm{s}_{1}, ..\right\}$. The eq. (3)., eq. (4)., and eq. (5). can be re-interpreted accordingly.

The success of the arms race for the different combatants will depend on their resources and agility as well as of explicit or implicit coalitions as indicated in eq. (5). The success of a strengthening of the protection system will for instance depend on the detection and reaction times for the user agent. A prevented virus attack is one example of a successful protection system. Spam mails and spy-wares are hard to fully protect against because there does not exist a crucial situation like in the virus situation, i.e., the expected loss $L$ of eq. (4). is not in general sufficient high to motivate an upgrading of the protection system.

The user's reaction against an exploiter measure could either be a direct response to the new measure or trying to anticipate future possibilities, including waiting for others to defeat the defense system (e.g., by using sniffing tools).

We can now state a robustness criterion. A robustness of the ecosystem, with respect to a protection system, of the ecosystem will emerge if and only if:

$$
\forall a \in \mathrm{A} \& \forall t^{\prime} \geq t, \exists s_{0}: \Delta E\left(a, s_{0}, t^{\prime}\right)<0
$$

where $A$ denotes all types of attacks and $t, t^{\prime}$ denotes points in time. If $\Delta E$ has small oscillations around zero the possibilities of reaching a robust state increases according to our formulas. It is the dynamics of the system, which influence the success of the infohabitants. Arms race will not destroy all intruders but make the system more robust, as in immunology. The lesson to learn is how to minimize the effect of intruders by finding almost stable robust states of the ecosystem.

\section{Discussion and Summary}

We propose a model of arms race between users and exploiters in a computational ecosystem. Exploiter agents try to, on the behalf of their human owners, make some extra profit (surplus) out of the ecosystem by exploiting user agents. The mechanisms generating surplus value is protected by a system implemented as a filter of access to the assets asked for. An arms race is modeled as a sequence of attacks and countermeasures strengthening the protection system. We have modeled the dynamics of the arms race by stating some basic formulas. The arms race might evolve in equilibrium of a stable robust system with respect to the protection mechanism under study.

Examples of protection systems include security and integrity systems in ebusiness. A typical attack in these systems includes spam mails. Spam mails direct advertising message to an unspecified group of receivers. A spam mail may look harmless compared to a virus. It does not cost the user any security harm or threats

CIA-finala submitted to CIA workshop : 2001-11-19: 09:41 
against stored data, but it costs time. A user agent filtering out spam mails costs processor time and delivered spam mails cost the user time for reading and cleaning up.

So, from a status quo perspective of the ecosystem, any changes involving some profit making is described as an attack, irrespective of if it is deleterious or evolve the information ecosystem.

There are two cases concerning the behavior of a protection system. A stable system or a dynamic system:

- A full secure stable system presupposes a total protection against every attack. The attacks must be detected and taken care of while the protection is still up. This ideal situation is rarely at hand. It could at best be attained as an emergent behavior.

- A dynamic system might have a strengthening process modeled as an arms race. An arms race presupposes some negative effects on the participants, but may not cause any immediate danger for the functionality of the system. A defense may include making anticipation against the intruder besides responding to the attack itself.

If all possible attacks are taken under consideration it is very unlikely that we can design a fully protected system. Instead we should expect an ecosystem based on arms race. The arms race is based on profit making activities. These activities make an improvement of the information ecosystem possible. A dynamic, evolving and robust ecosystem of autonomous agents is a preferred and possible outcome of the arms race.

The reported work is preliminary in many respects. We are at present evaluating our model in arms race scenarios based on Napster and other peer-to-peer ecosystems emerging on the Internet.

\section{References}

1. Carlsson, B. and Davidsson, P. A Biological View on Open Agent Systems to appear in IAT'2001, Maebashi City, Japan (2001)

2. Dawkins, R.: The Selfish Gene. 2nd edn. Oxford University Press, Oxford (1989)

3. Durfee, E., Lesser,V., and Corkill,D., Cooperative distributed problem solving, in ed. Barr,A., Cohen,P., and Feigenbaum, E., The Handbook of Artificial Intelligence, volume IV, p. 83-147, Addison Wesley (1989)

4. Durfee, E.H. Coordination of distributed problem solvers Kluwer (1988)

5. Gustavsson, R.: Security Issues and Power Line Communication. Invited Key address. In Proceedings of the Fifth International Symposium on Power Line Communication, (2001)

6. Jennings, N.R., Commitments and Conventions: The foundation of Coordination in MultiAgent Systems, The Knowledge Engineering Review, 2 (3) p. 223-250, (1993)

7. Maynard Smith, J., Evolution and the theory of games, Cambridge Univ. Press, (1982)

8. Sandholm,T.W., and Lesser, V.R., Coalitions among computationally bounded agents, Artificial Intelligence, 94(1) p. 99-137 (1997)

9. Schwartau W. Time Based Security. Practical and Provable Methods to Protect Enterprise and Infrastructure, Networks and Nation Interpact Press (1999)

10. Wellman, M., A computational market model for distributed configuration design, Proc. $12^{\text {th }}$ National Conference on Artificial Intelligence (AAAI-94) p. 401-407, Seattle, WA (1994)

11. Wilson, E.O.: Sociobiology - The abridged edition. Belknap Press, Cambridge (1980) 